\title{
Craniosynostosis : Updates in Radiologic Diagnosis
}

\author{
Hyun Jeong Kim, M.D., Ph.D., ${ }^{1}$ Hong Gee Roh, M.D., Ph.D., ${ }^{2}$ II Woo Lee, M.D., Ph.D. ${ }^{3}$ \\ Departments of Radiology, ${ }^{1}$ Neurosurgery, ${ }^{3}$ Daejeon St. Mary's Hospital, The Catholic University of Korea, Daejeon, Korea \\ Department of Radiology, ${ }^{2}$ Konkuk University Medical Center, Konkuk University School of Medicine, Seoul, Korea
}

The purpose of this article is to review imaging findings and to discuss the optimal imaging methods for craniosynostosis. The discussion of imaging findings are focused on ultrasonography, plain radiography, magnetic resonance imaging and computed tomography with 3-dimensional reconstruction. We suggest a strategy for imaging work-up for the diagnosis, treatment planning and follow-up to minimize or avoid ionized radiation exposure to children by reviewing the current literature.

Key Words : Skull · Cranial sutures · Craniosynostosis · Computed tomography · Plain radiograph · Magnetic resonance imaging · Ultrasonography.

\section{INTRODUCTION}

Craniosynostosis is a condition of the premature fusion of one or more cranial sutures, which results in characteristic skull shape deformities and facial asymmetry accompanied by functional consequences such as increased intracranial pressure (ICP), visual impairment, deafness and cognitive deficits ${ }^{4,11,12,14,18)}$. Craniosynostosis can occur primarily and secondarily. Premature fusion of one or more of the sutures is believed to be due to a developmental error during embryogenesis in primary craniosynostosis whereas secondary craniosynostosis is due to mechanical causes such as intrauterine compression of the fetal skull, metabolic causes, and the effect of teratogens. Approximately $85 \%$ of primary craniosynostosis cases occur as an isolated condition and the remaining $15 \%$ are a part of a multisystem syndrome ${ }^{10,13)}$.

Imaging is essential for the accurate diagnosis, surgical planning, post-treatment evaluation and identification of coexisting anomalies and complications associated with craniosynostosis. Because of its superior bone depiction, computed tomography (CT) with three-dimensional (3-D) reconstruction is the primary imaging technique used in craniosynostosis. However, exposure to ionized radiation from CT scans, especially in infants, has led researchers to explore alternative imaging techniques and protocols for craniosynostosis including the use of ultrasonography, plain skull radiography and magnetic resonance imaging (MRI). Here we present imaging findings in pa- tients with normal craniums, craniosynostosis and its mimics and discuss the optimal imaging technique for the diagnosis, treatment planning and follow-up of craniosynostosis.

\section{REVIEW OF IMAGING FINDINGS OF NORMAL CRANIAL SUTURES AND CRANIOSYNOSTOSIS}

\section{Imaging findings of normal cranial sutures}

Cranial sutures exist along the margins of adjacent paired cranial bones, namely the frontal, parietal, temporal and occipital bones. Specifically, the metopic suture is between the frontal bones; the sagittal suture is between the parietal bones; the coronal sutures are between the frontal and parietal bones; the lambdoid sutures are between the parietal and occipital bones; and the squamosal sutures are between the parietal and temporal bones. Furthermore, the transverse occipital suture is between the squamous and supraoccipital segments of the occipital bone, which fuses in its midportion and forms the paired lateral mendosal sutures (Fig. 1) ${ }^{1}$. The fontanels are formed at the junctional boundaries of the cranial sutures. The cranial sutures in neonates appear wide open and fuse with age from back to front and lateral to medial with the exception of the metopic suture, which fuses from front to back. Normally the metopic suture fuses between 9 months and 2 years of age, followed by the coronal, lambdoid and then the sagittal sutures. These sutures fuse and disappear between 30 and 40 years of age as the growth of the cranial bones at the sagittal, coronal

• Received : January 22, 2016 • Revised : April 21, 2016 • Accepted : April 22, 2016

- Address for reprints : II Woo Lee, M.D., Ph.D.

Department of Neurosurgery, Daejeon St. Mary's Hospital, The Catholic University of Korea, 64 Daeheung-ro, Jung-gu, Daejeon 34943, Korea

Tel : +82-42-220-9638, Fax : +82-42-220-9087, E-mail : leeilwoo@catholic.ac.kr

- This is an Open Access article distributed under the terms of the Creative Commons Attribution Non-Commercial License (http://creativecommons.org/licenses/by-nc/3.0) which permits unrestricted non-commercial use, distribution, and reproduction in any medium, provided the original work is properly cited. 
and lambdoid sutures ceases early in the second decade of life. The anterior fontanel fuses at approximately 18 months of age, and the posterior fontanel fuses between 3-6 months of age ${ }^{1,2,10,13)}$.

Normal skulls of children show variable appearances in the initial days and months after birth because brain growth accompanied with changes in the cranial bones and sutures is explosive during the first 2 years of life (Fig. 1). A normal patent suture is demonstrated as a radiolucent, serrated and nonlinear line on plain skull radiography and 3-D CT images (Fig. $1)^{1,2,10,13)}$. Ultrasonography shows a normal patent suture as an uninterrupted hypoechoic fibrous gap between hyperechoic cranial bones with an end-to-end appearance on a transverse scan of the sagittal sinus and a beveled appearance on a trans- verse scan of the coronal and lambdoid sutures (Fig. 2) ${ }^{15,19,20)}$. Conventional MRI has typically been unreliable in identifying sutures individually. However, recently Eley et al. ${ }^{6}$ described a novel gradient echo MRI sequence ("Black Bone") that minimizes soft tissue contrast to enhance the bone-soft tissue boundaries and can demonstrate normal patent cranial sutures as hyperintensity distinguished from the signal void of the cranial bones (Fig. 3A).

\section{Imaging findings of craniosynostosis}

Premature fusion of the cranial sutures restricts cranial growth perpendicular to the affected suture with compensatory overgrowth along the other patent sutures. This results in the char-
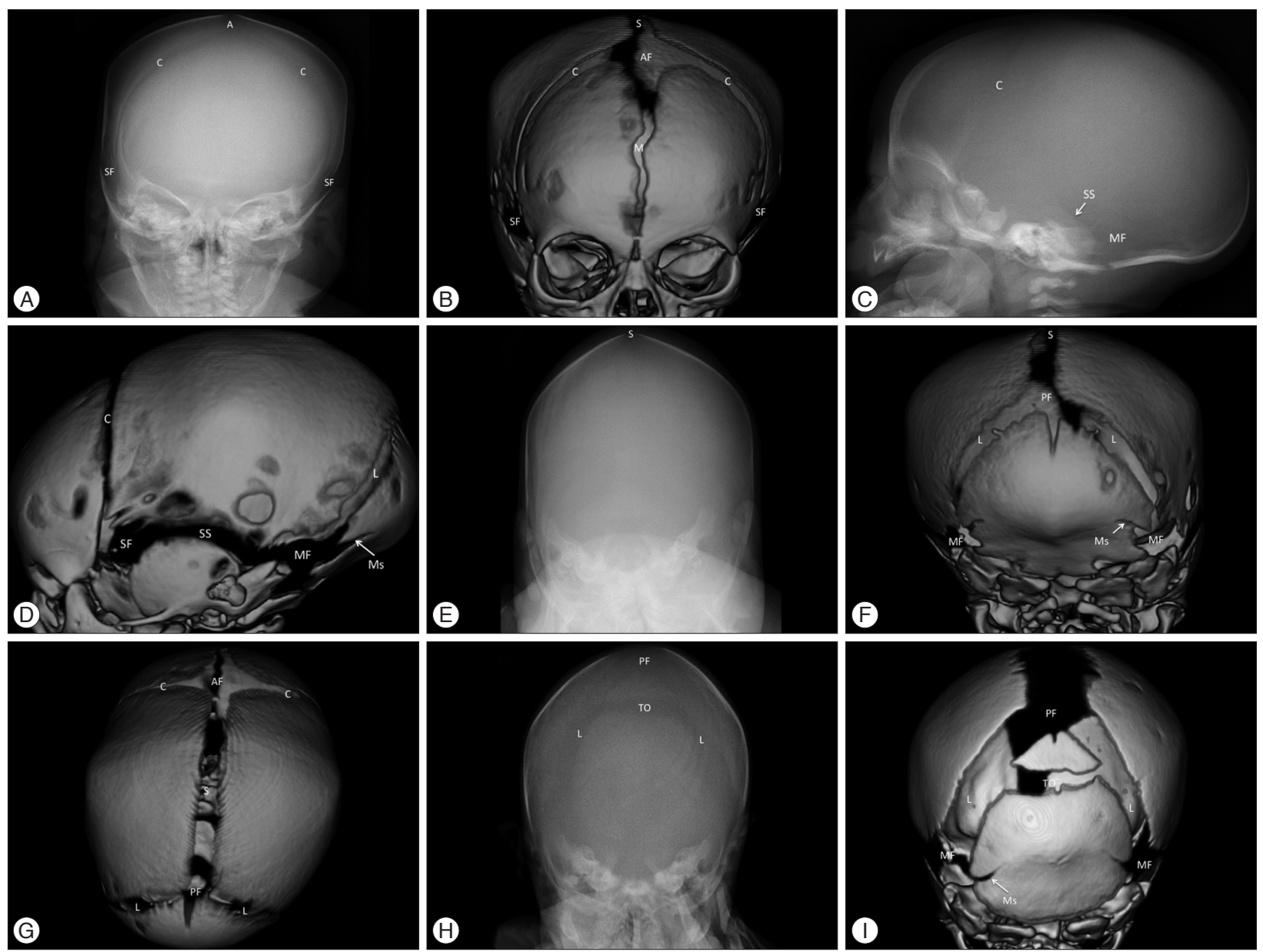

Fig. 1. Images of the normal anatomy of the cranial sutures of two neonates, 2 days $(A-G)$ and 19 days $(H$ and $I)$ of age, and an infant at 12 months $(\mathrm{J}-\mathrm{N})$ of age using plain skull radiography and 3-dimensional (3-D) computed tomography (CT). Plain skull radiography images of the anterior-posterior (AP), lateral and posterior-anterior (PA) views are paired with 3-D CT images of the same direction. A and B are AP view images using plain skull radiography and 3-D CT, respectively; $\mathrm{C}$ and $\mathrm{D}$ are lateral view images; $\mathrm{E}$ and $\mathrm{F}$ are PA view images of a neonate at 2 days of age; $\mathrm{H}$ and I are PA images of a neonate at 19 days of age; and J-N are images of a 12-month-old boy. The superior view images (G and N) show only the 3-D CT images. Normal metopic (M), coronal (C), sagittal (S), lambdoid (L), mendosal (Ms), transverse occipital (TO), and squamosal (SS) sutures are demonstrated with a radiolucent, serrated and nonlinear appearance on plain and CT images. The anterior (AF), posterior (PF), sphenoidal (SF), and mastoid (MF) fontanels are also shown as radiolucent spaces. The typical radiolucent and serrated sutures become more distinct with age (comparing the images of the 2-day-old neonate with the images of the 12-month-old infant). The partially fused metopic suture, open AF and closed PF are normal at 12 months of age $(\mathrm{J}, \mathrm{K}$, and $\mathrm{N})$. The cranial sutures of neonates have substantial variability. The distances between the bone plates of two the neonates are quite different normally ( $\mathrm{F}$ and $\mathrm{I})$, and the transverse occipital suture may or may not be seen in neonates ( $\mathrm{F}$ and $\mathrm{I})$. Neonatal sutures can be indistinct on plain skull radiography until 3 months after birth due to low thickness and low mineralization of the cranial bone, which impedes proper judgment of the state of the sutures. The metopic suture in $A$ and the lambdoid sutures in $C$ and $E$ are indistinct in the lateral and PA images of the plain skull radiography. However, they are clearly demonstrated as patent sutures in the CT images (B, D, and F).

(Continued to the next page) 

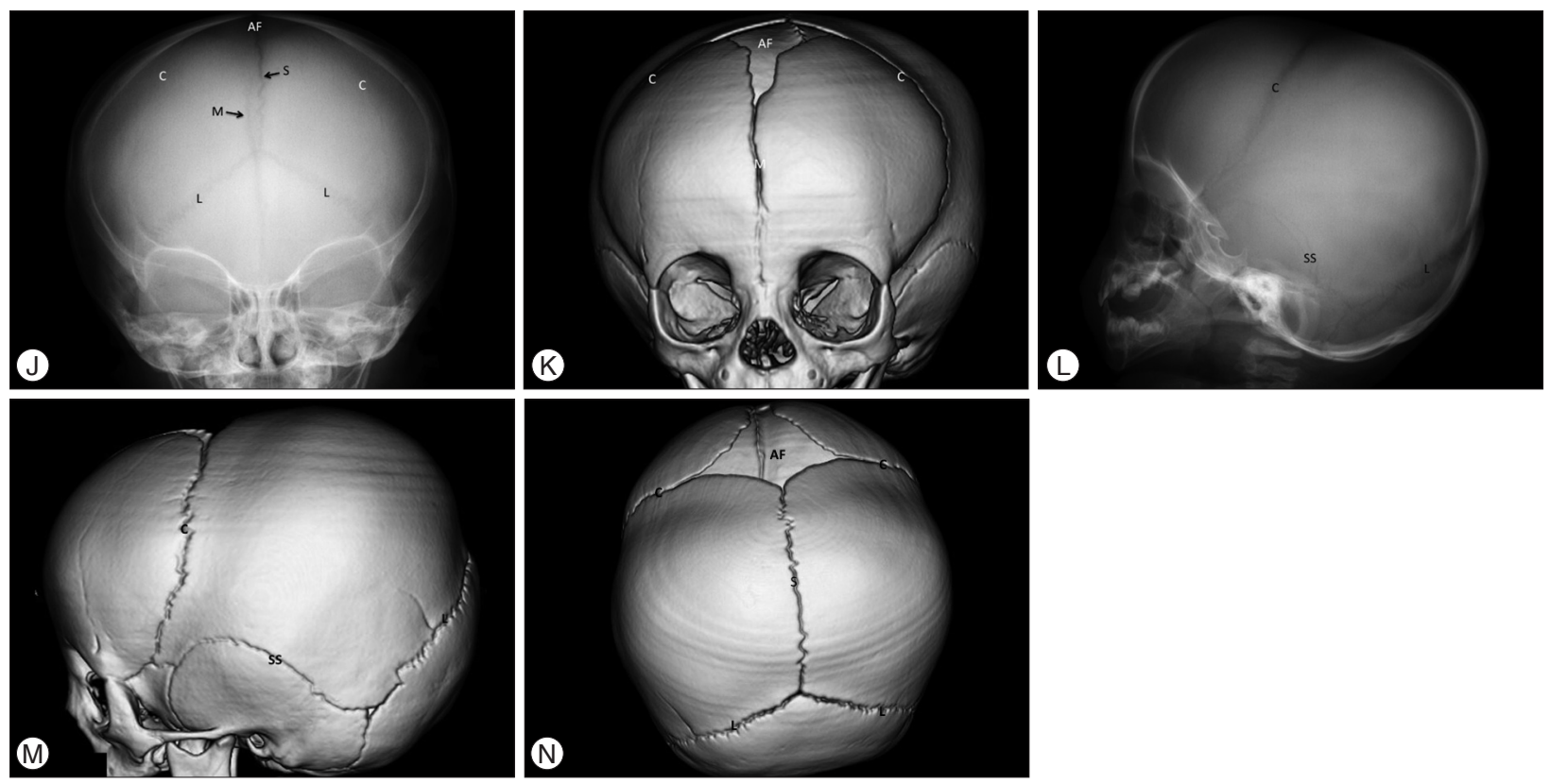

Fig. 1. (Continued fom previous page) Images of the normal anatomy of the cranial sutures of two neonates, 2 days $(A-G)$ and 19 days $(H$ and $I)$ of age, and an infant at 12 months $(\mathrm{J}-\mathrm{N})$ of age using plain skull radiography and 3-dimensional (3-D) computed tomography (CT).
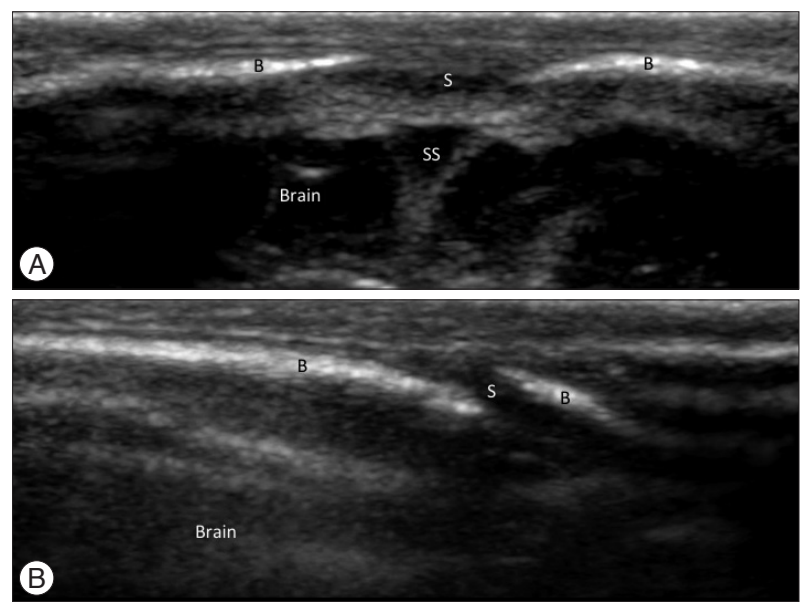

Fig. 2. Ultrasonographic findings of normal cranial sutures of a 2-day-old boy. A : A transverse sonogram of the sagittal suture shows the suture as a hypoechoic gap (S) between hyperechoic cranial bones (B) with end-to-end appearance. The superior sagittal sinus (SS) and the brain are noted under the skull. $\mathrm{B}: \mathrm{A}$ transverse sonogram of the lambdoid suture shows the suture as a hypoechoic gap (S) between hyperechoic cranial bones $(B)$ with a beveled appearance. This finding is also noted on ultrasonography of the coronal suture.

acteristic skull shape deformities noted in craniosynostosis. Diagnostic imaging is necessary to confirm the fused suture and to assess the accompanying skull deformities, intracranial pathology and other complications. A prematurely fused suture shows perisutural sclerosis, linearity, reduced serration, bony bridging or the absence of the suture on a plain skull radiography or CT image. Secondary signs of increased ICP, such as a "copper-beaten" appearance, are also observed in severe cases. Soboleski et al..$^{20)}$ reported the ultrasonographic findings of craniosynostosis as follows : 1) the loss of the hypoechoic fibrous gap between hyperechoic body plates; 2) an irregular, thickened inner sutural margin; 3) the loss of a beveled edge; and 4) asymmetric fontanels. On "Black Bone" MRI, the affected fused sutures are demonstrated as absence of suture (Fig. 3B $)^{6}$.

\section{Sagittal synostosis}

Restriction of growth perpendicular to the sagittal suture results in a narrow and elongated cranium with frontal bossing, occipital protrusion and ridging of the fused, heaped suture. This deformity is characterized by scaphocephaly (inverted boat with a keel), clinocephaly (flat cranium due to loss of cranial convexity), and leptocephaly (tall and narrow cranium) (Fig. 4).

\section{Coronal synostosis}

Coronal synostosis causes restriction of growth in the anterior-posterior direction, perpendicular to the coronal suture with compensatory overgrowth in the parietal direction, perpendicular to the patent sagittal suture. Unicoronal synostosis results in anterior plagiocephaly (oblique cranium) and appears as a shallow ipsilateral anterior cranial fossa with contralateral frontal bossing, an elevated roof and lateral wall of the ipsilateral orbit ("harlequin appearance"), a hypoplastic supraorbital ridge, elevated ipsilateral sphenoid wings, a contralaterally shifted anterior fontanel and facial asymmetry (Fig. 5). Bicoronal synostosis restricts skull growth in the anterior-posterior direction, which generally results in brachycephaly (short cranium). Isolated bicoronal synostois is relatively rare but is commonly observed in syndromic synostosis and is associated with upper and midface hypoplasia along with other craniofacial deformities and premature fusion of the skull base sutures leading to a small posterior fossa. Images reveal fused bicoronal sutures, 

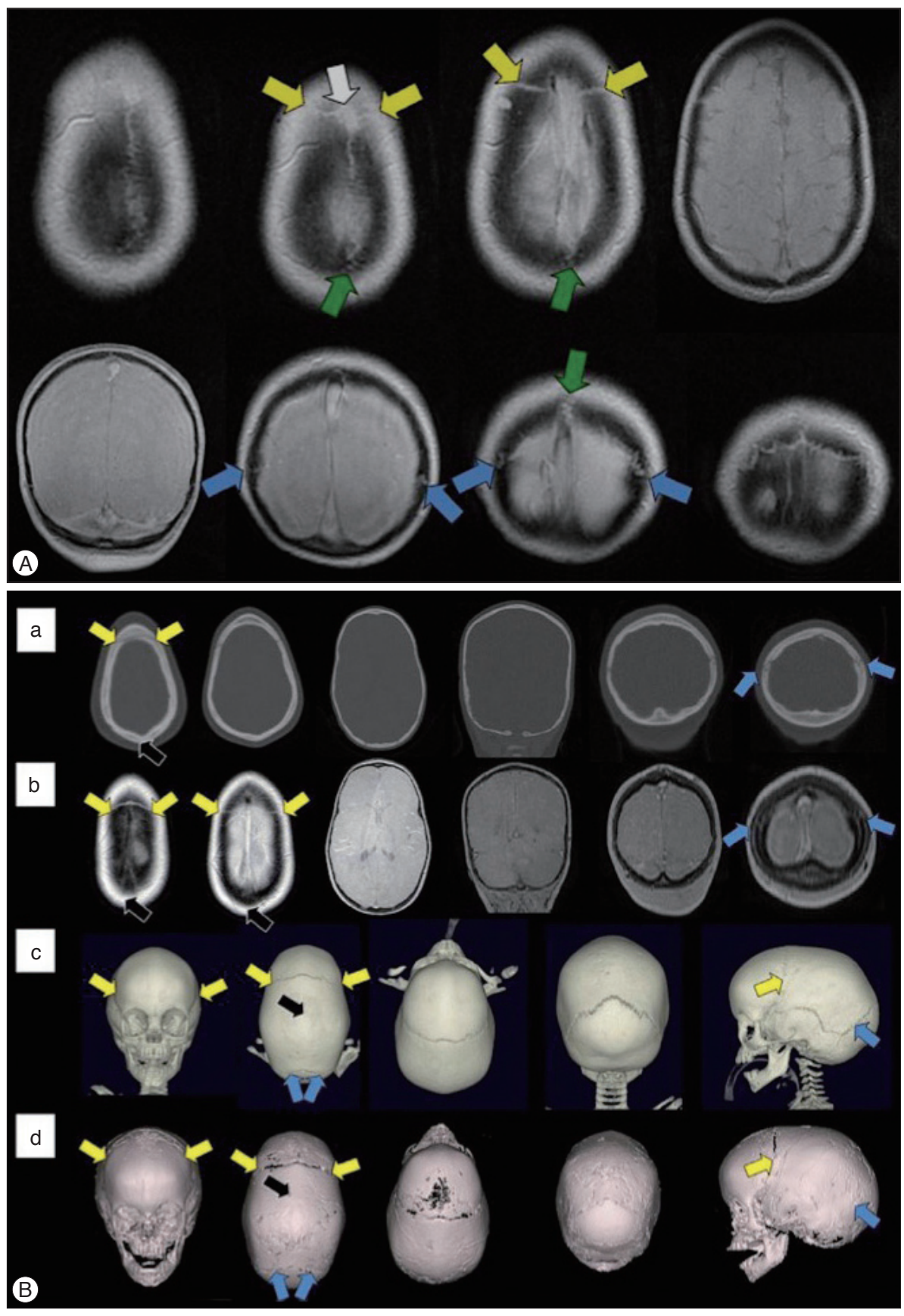

Fig. 3. Magnetic resonance imaging (MRI) findings of normal cranial sutures and craniosynostosis of children. A : Axial (top row) and coronal (bottom row) "Black Bone" imaging show normal patent cranial sutures as hyperintensity distinguished from the signal void of the cranial bones (Coronal suture : yellow arrow; Lambdoid suture : blue arrow; Sagittal suture : green arrow, visible anterior fontanelle : white arrow). B : Images of axial and coronal CT (a), axial and coronal "Black Bone" MRI (b), 3-D CT (c), and 3-D "Black Bone" MRI (d) show sagittal synostosis (Coronal suture : yellow arrow; Lambdoid suture : blue arrow; the site of synostosis : black arrow). Adapted from Eley KA, et al., Eur Radiol 24 : 2417-2426, 2014 ${ }^{6}$ with permission from Springer.

shortened anterior-posterior and widened biparietal dimensions of skull, and bilateral "harlequin eye" deformity of the orbits with increased interorbital distance (hypertelorism) (Fig. 6).

\section{Metopic synostosis}

Premature fusion of the metopic suture results in a palpable ectocranial ridge or omega-shaped notch and a small anterior cranial fossa with a triangular pointed forehead (trigonocephaly) and parieto-occipital bossing due to bilateral constriction of the frontal bones. Imaging findings include decreased interorbital distance (hypotelorism), hypoplastic ethmoid sinus, and medially upward slanted orbital roof ("quizzical eye" appearance)
(Fig. 7). Metopic synostosis should be differentiated from the metopic ridge, which is a normal variant of the metopic suture closure. One-third of cases of metopic synostosis are syndromic, with associated midline anomalies of the brain and the palate.

\section{Lambdoid synostosis}

A unilateral premature fusion causes posterior plagiocephaly (oblique deformity of the posterior cranium), which is more commonly caused by positional problems compared to premature fusion of the cranial suture. Therefore, unilateral lambdoid synostosis should be differentiated from positional plagiocephaly. Images show ipsilateral occipitoparietal flattening, contra- 

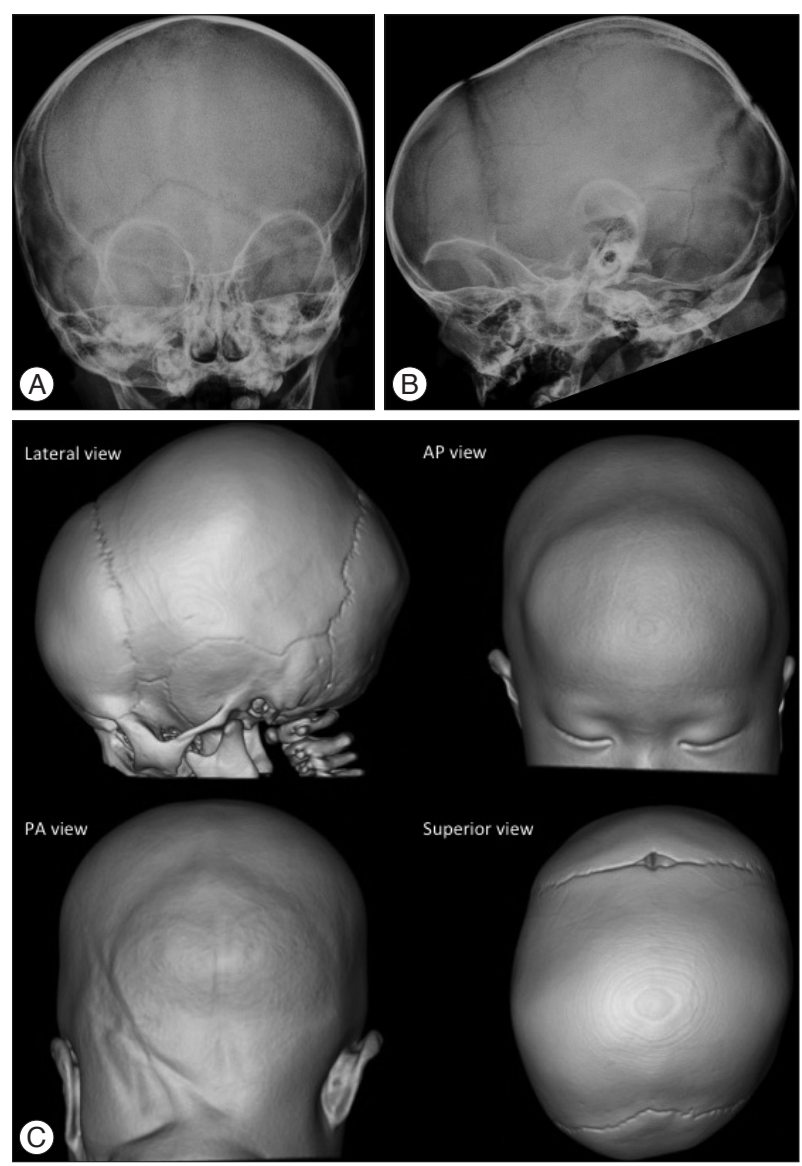

Fig. 4. Sagittal synostosis in an 11-month-old boy. The plain skull radiography (A : AP view, B : Lateral view) and 3-D CT images (C) show the absence of a radiolucent sagittal suture ( $A$ and $C$ ) and an elongated cranium with frontal bossing and occipital protrusion (B and $\mathrm{C}$ ). The patent coronal and lambdoid sutures have a radiolucent, serrated and nonlinear appearance, and the fused metopic suture is normal in this 11-month-old baby.

lateral frontal and occipitoparietal bossing, a contralaterally shifted posterior fontanel, double contouring of the occipital margin due to a unilateral small posterior cranium, and a tilted skull base (Fig. 8). Bilateral fusion of the lambdoid sutures results in turricephaly (tall cranium; also known as oxycephaly and acrocephaly), with bilateral occipitoparietal flattening that leads to a small posterior cranial fossa and unimpeded compensatory growth at bregma.

\section{Multisuture synostosis}

Multisuture synostosis is often syndromic. The patterns are variable according to the combination of sutures. Pansynostosis involves the sagittal, coronal and lambdoid sutures and is the most severe form resulting in the cloverleaf deformity of the skull (bulging of the temporal regions and severe proptosis, also known as Kleeblattschäde) or oxycephaly (a narrow and towering cranium) (Fig. 9). Bilateral lambdoid and sagittal synostosis is called Mercedes Benz synostosis. These complex forms of synostosis are often combined with other structural and functional complications.
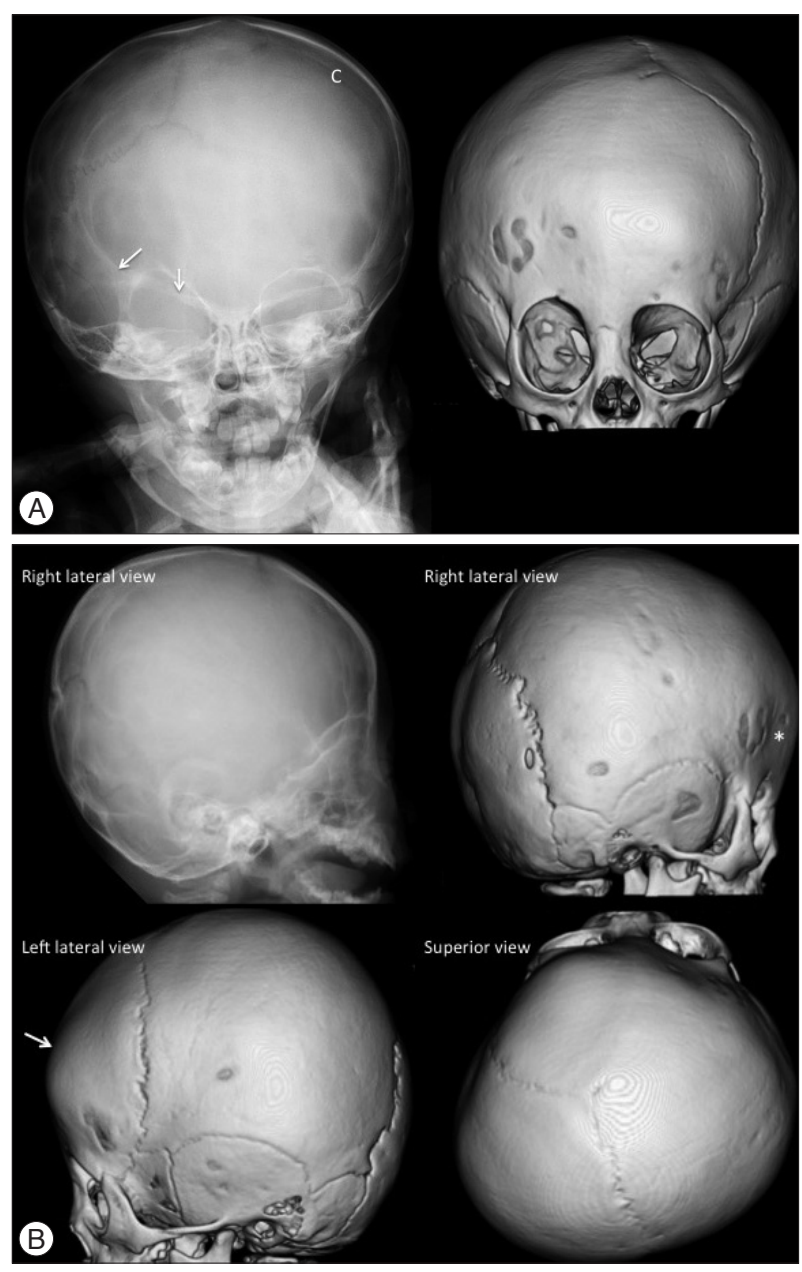

Fig. 5. Unicoronal (right) synostosis in a 12-month-old girl. A : The plain skull radiography and 3-D CT AP view images show the fused right coronal suture as the absence of a suture and a bony ridge compared to the patent left coronal suture (C). The "harlequin eye" appearance of the right orbit, the elevated ipsilateral sphenoid wings (arrows), the facial asymmetry (deviation of the nose and chin to the contralateral side), and the contralaterally shifted anterior fontanel are also demonstrated. The 3-D CT images also show the contralateral frontal bossing and ipsilateral hypoplastic supraorbital ridge. B : The plain skull radiography and 3-D CT lateral and superior view images show the absence of the right coronal suture, shallow ipsilateral anterior cranial fossa (*) and contralateral frontal bossing (arrow). Anterior plagiocephaly due to the decreased AP dimension of the right cranium with shifted anterior fontanel and contralateral frontal bossing are demonstrated on the superior view image.

\section{Imaging findings of craniosynostosis mimics}

\section{Metopic ridge}

Metopic ridge is a normal variant of the metopic closure that occurs in $4 \%$ of asymptomatic children between $0-18$ months of age and should be differentiated from metopic synostosis. Infants with metopic ridge show a fused metopic suture without the other characteristic features of metopic synostosis such as trigonocephaly, hypotelorism, and the "quizzical eye" appearance ${ }^{23}$.

\section{Deformational plagiocephaly}

Positional deformities arise in infants from a preferential 
head positioning on one side during sleep and results in asymmetrical occipital flattening (posterior plagiocephaly). This is amenable to conservative treatment contrary to lambdoid synostosis, which requires surgical correction. The lambdoid suture in deformational plagiocephaly is patent and ipsilateral frontal bossing is combined as opposed to lambdoid synostosis (Fig. 10 compare with Fig. 8).

\section{DISCUSSION FOR OPTIMAL IMAGING FOR DIAGNOSIS AND FOLLOW-UP OF CRANIOSYNOSTOSIS}

Although 3-D CT has superior diagnostic value, concerns about the hazards of radiation exposure in children has led to a consensus to avoid or postpone radiographic CT imaging until the end of the first year of life or as late as possible in children with suspected or diagnosed craniosynostosis ${ }^{1,15-17)}$. The first

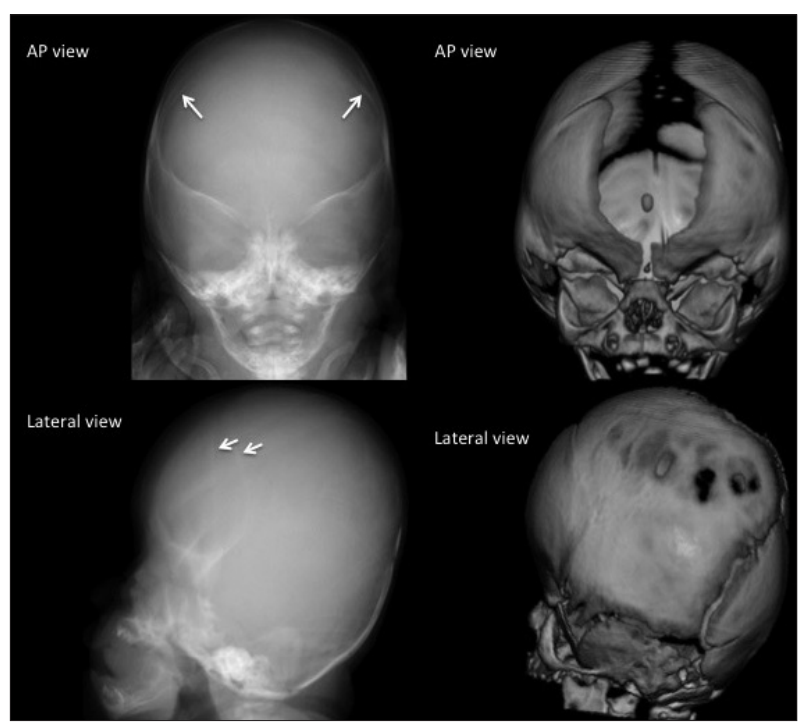

Fig. 6. Bicoronal synostosis in a 1-month-old boy. The fusion of both coronal sutures is demonstrated as sclerotic linear ridges (arrows). The patent metopic suture and anterior fontanel in this 1- month-old baby are normal. The plain radiography of this 1-month-baby does not show the wide open metopic suture. However, it demonstrates the sclerotic fused suture. Brachycephaly (short cranium by shortened AP and widened biparietal dimensions) and the bilateral "harlequin eye" deformity of the orbits combined with hypertelorism are characteristic of bicoronal synostosis. step in assessing patients with an abnormal skull shape is a clinical examination in which almost all forms of monosutural cra-
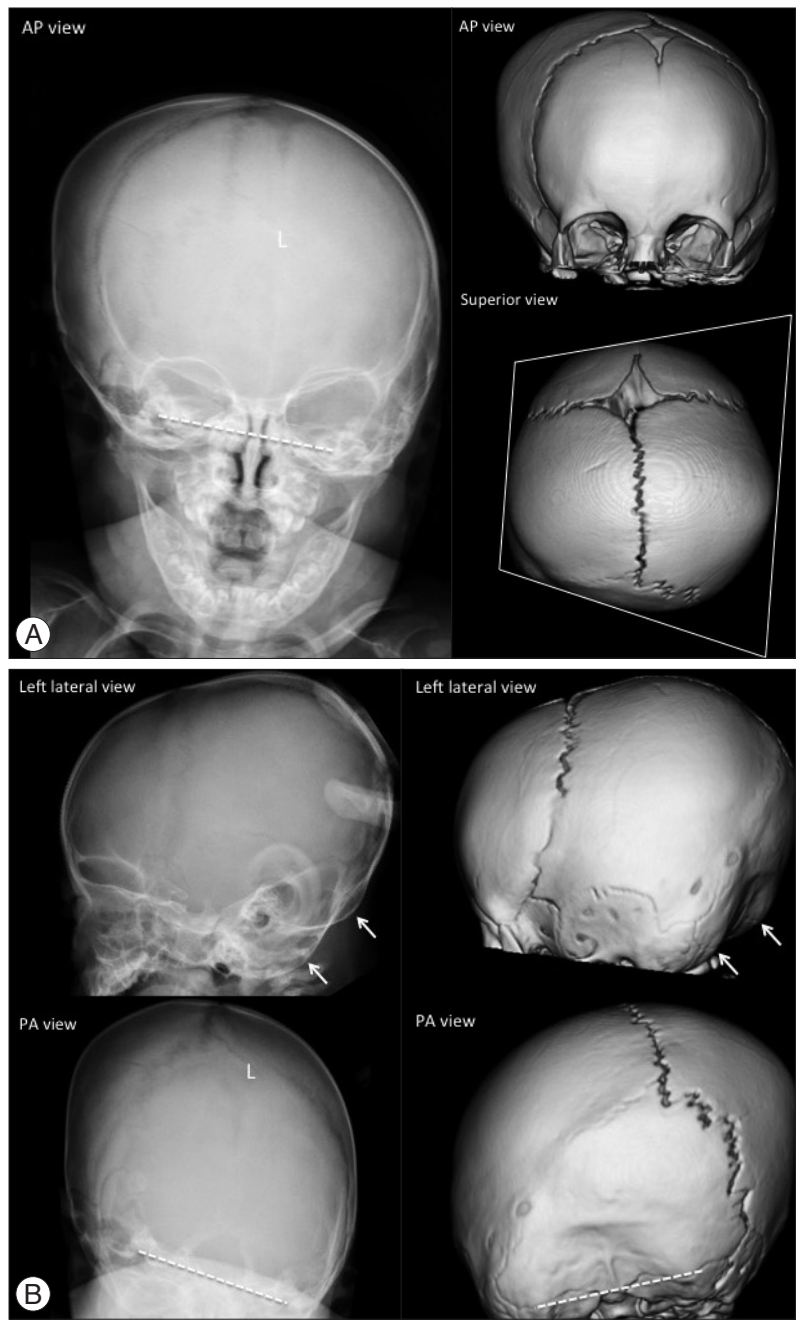

Fig. 8. Unilateral (left) lambdoid synostosis in an 8-month-old boy. The plain skull radiography and 3-D CT images show the fused suture as a partial absence of the suture with a linear ridge and perisutural sclerosis ( $\mathrm{L}$ in $\mathrm{A}$ and $\mathrm{B}$ ). Ipsilateral occipitoparietal flattening (posterior plagiocephaly), contralateral frontal and occipitoparietal bossing, and contralaterally shifted posterior fontanel are demonstrated. The cranium shows a trapezoidal configuration on the superior view image. The unilateral small posterior cranium makes a double contour of the occipital margin on the lateral view images (arrows in B), and the tilted skull base is noted in the $A P$ and $P A$ view images (dashed lines in $A$ and $B$ ).

Fig. 7. Metopic synostosis in a 12-monthold boy. A fused metopic suture is demonstrated as a linear sclerotic line on the plain radiography image and a bony ridge on the 3-D CT image (arrows). Trigonocephaly, hypotelorism, a small ethmoid sinus, and the "quizzical eye" appearance of the orbits are noted.

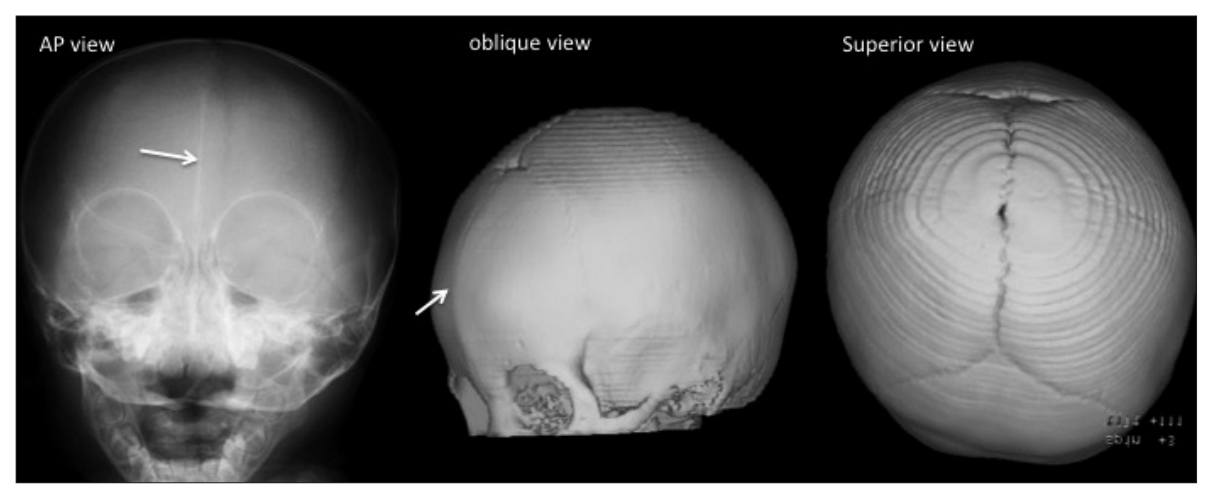



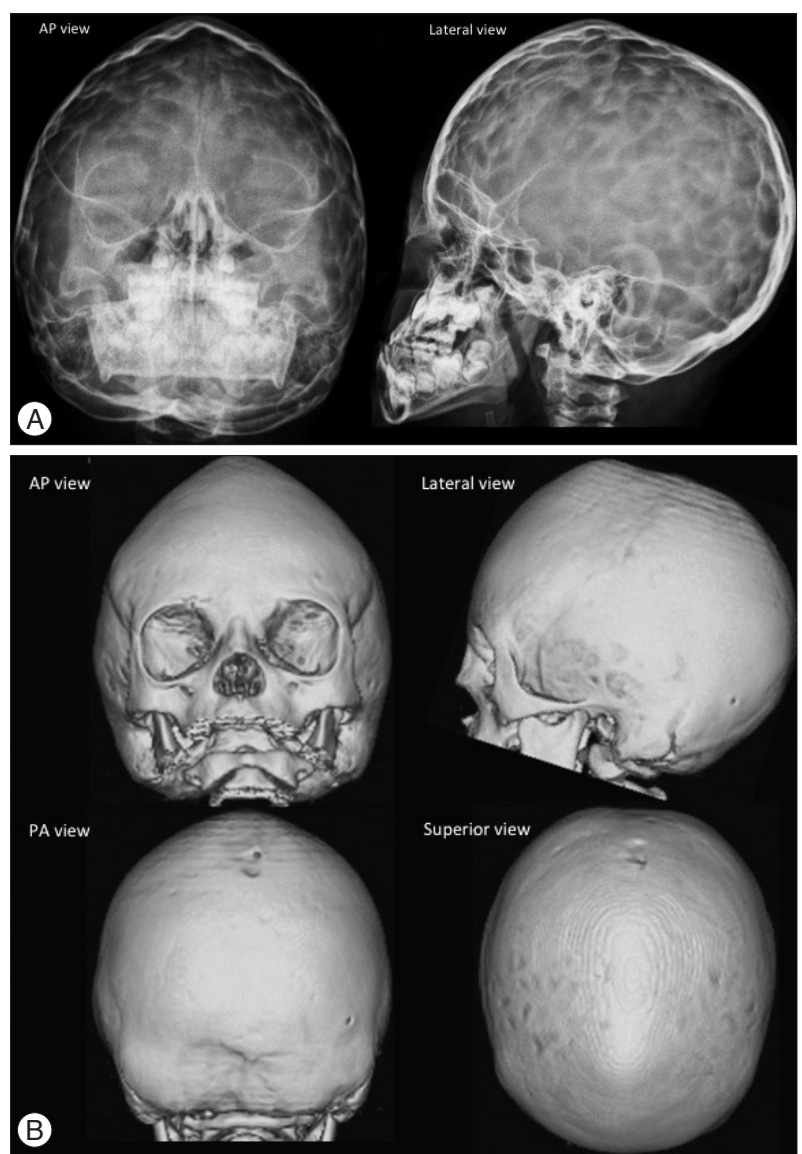

Fig. 9. Pansynostosis in a 10-month-old girl with Crouzon syndrome. The plain skull radiography (A) and 3-D CT (B) images show an absence of the coronal, sagittal and lambdoid sutures. The fontanels are also closed. The cranium is narrow and towered (oxycephaly). "Copper beaten" skull is also demonstrated on the plain radiography images (A) by gyral impressions on the inner table of the skull, which represents the increased intracranial pressure. niosynostoses can be diagnosed. If there is a question of the diagnosis, ultrasonography is the technique of choice for diagnosis of neonates and infants. This simple and radiation-free technique can differentiate fused or patent sutures effectively and differentiate nonsynostotic pathology, such as positional skull deformities and molding, from craniosynostosis ${ }^{20,21)}$. Increase of the width of the cranial sutures on serial sonograms may provide evidence for a possible increase in ICP, which is a major complication of craniosynostosis ${ }^{20)}$. Schweitzer et al. ${ }^{17)}$ investigated the individual features of all forms of craniosynostosis in plain skull radiography (AP and lateral images) to achieve proper diagnosis while avoiding the use of a CT scan. The effective radiation dose of plain skull radiography is approximately $0.01 \mathrm{mSv}$ to $0.04 \mathrm{mSv}$. In monosutural craniosynostosis, plain skull radiography is only indicated in children with remaining diagnostic doubts after clinical and sonographic evaluation, immediately prior to surgery or in non-surgical cases after the first year of life to access for additionally affected sutures ${ }^{16)}$. A CT scan may be unavoidable in complex (multisutural and syndromic types) or complicated types of craniosynostosis for treatment planning. The reported effective dose of radiation for a 3 -D CT scan is in the range of $0.2 \mathrm{mSV}$ to $2 \mathrm{mSV}^{3,5,8,22)}$. Recently, Ernst et al. ${ }^{7)}$ reported that a $0.08 \mathrm{mSv} 3-\mathrm{D}$ CT protocol by using Model Based Iterative Reconstruction can be used in children with cranial deformities without compromising imaging quality. In a phantom study, Kaasalainen et al.9) achieved sufficient image quality using a CT protocol with an effective radiation dose of $0.02 \mathrm{mSv}$, which is comparable to the effective dose of plain skull radiography. Although this ultra-low-dose CT technique is not widely available presently and the diagnostic value for minor sutures is still questionable, these results are promising in children with complex craniofacial malformations who
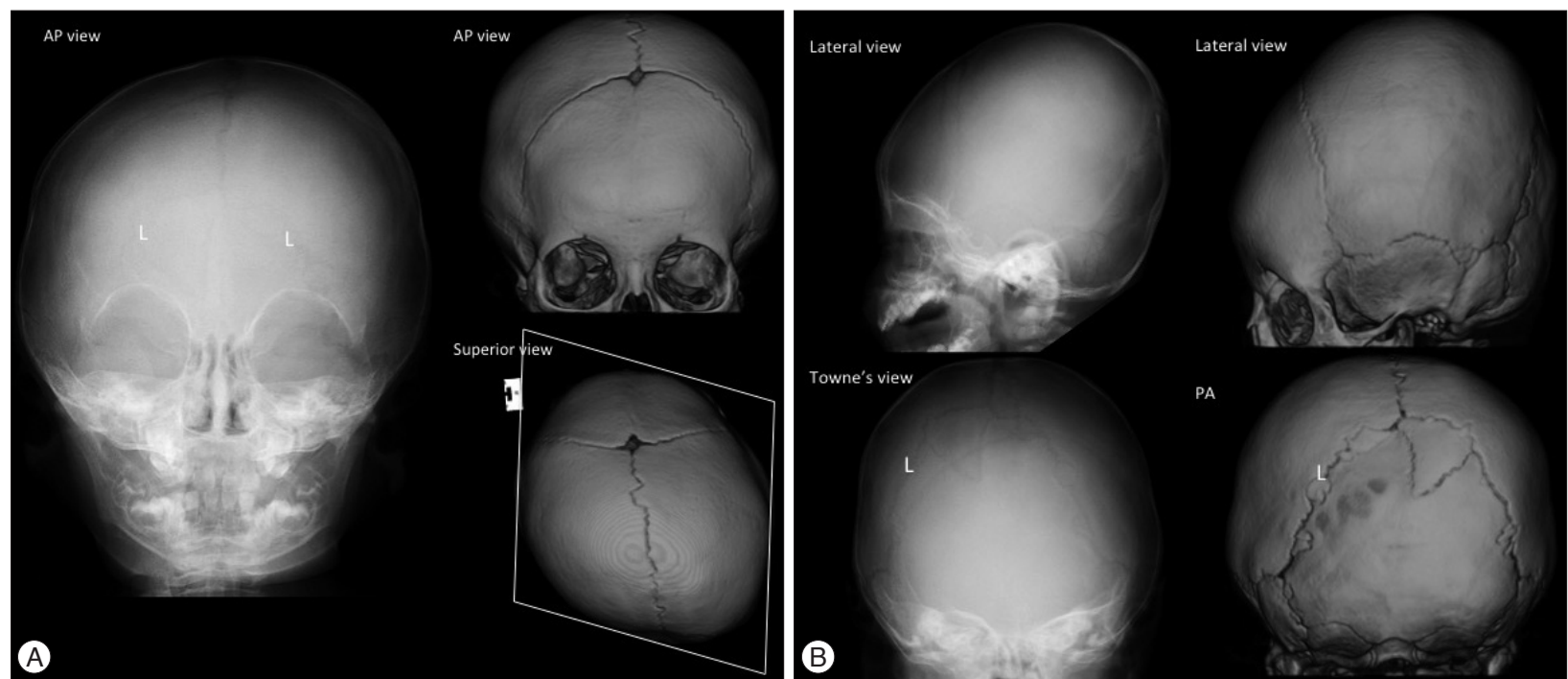

Fig. 10. Deformational plagiocephaly in a 10-month-old girl. Posterior plagiocephaly is seen on a superior view image of a 3-D CT due to left occipitoparietal flattening. However, both the lambdoid sutures $(\mathrm{L})$ are patent with normal appearance and ipsilateral frontal bossing contrary to plagiocephaly caused by lambdoid craniosynostosis (A and B). The cranium shows a parallelogram configuration on the superior view image (A). The double contour of the occipital margin and the tilted skull based are not noted in deformational plagiocephaly (B). 
undergo repeated CT scans at the time of diagnosis and at various stages of surgical correction. The role of MRI in craniosynostosis has been limited due to its unreliability in identifying the cranial sutures. However, with advantages of radiation-free and excellent soft-tissue contrast, MRI has been an important modality in combination with ultrasonography in infants with suspected associated intracranial anomalies and complications of craniosynostosis. As mentioned above, Eley et al. ${ }^{6}$ established a novel 3-D MRI technique ("Black Bone") that is able to individually identify the cranial sutures similar to other 2-D imaging modalities. Further research is necessary to standardize the use of 3-D MRI's of the cranium; however, this technique is revolutionary for radiosensitive infants with craniosynostosis.

With advancements in devices and technology, we can presently apply MRI and $0.2 \mathrm{mSv} 3-\mathrm{D}$ CT to inspect the cranial bones, sutures and soft tissue of children with craniosynostosis. However, clinical evaluation followed by ultrasonography should be considered the first step for imaging diagnosis in infants with craniosynostosis. MRI should be considered in children with suspected intracranial anomalies and complications. Plain skull radiography satisfies the clinical need in the majority cases involving monosutural craniosynostosis, even for preoperative planning of surgical correction and postoperative followup. CT should be reserved for children with complex craniofacial deformities to plan the management of the disease. Presently, available commercial low-dose 3-D CT scanners have an effective radiation dose of approximately $0.2 \mathrm{mSv}$. In addition, 3-D MRI and ultra-low-dose 3-D CT scanners are expected as an alternative to plain skull radiography in the near future.

\section{References}

1. Badve CA, K MM, Iyer RS, Ishak GE, Khanna PC : Craniosynostosis : imaging review and primer on computed tomography. Pediatr Radiol 43 : 728-742; quiz 725-727, 2013

2. Branson HM, Shroff MM : Craniosynostosis and 3-dimensional computed tomography. Semin Ultrasound CT MR 32 : 569-577, 2011

3. Calandrelli R, D’Apolito G, Gaudino S, Sciandra MC, Caldarelli M, Colosimo C: Identification of skull base sutures and craniofacial anomalies in children with craniosynostosis : utility of multidetector CT. Radiol Med 119: 694-704, 2014

4. Cohen MM Jr : Craniosynostosis and syndromes with craniosynostosis : incidence, genetics, penetrance, variability, and new syndrome updating. Birth Defects Orig Artic Ser 15 : 13-63, 1979

5. Didier RA, Kuang AA, Schwartz DL, Selden NR, Stevens DM, Bardo DM : Decreasing the effective radiation dose in pediatric craniofacial CT by changing head position. Pediatr Radiol 40 : 1910-1917, 2010

6. Eley KA, Watt-Smith SR, Sheerin F, Golding SJ : "Black Bone" MRI : a potential alternative to $\mathrm{CT}$ with three-dimensional reconstruction of the craniofacial skeleton in the diagnosis of craniosynostosis. Eur Radiol 24 : 2417-2426, 2014

7. Ernst CW, Hulstaert TL, Belsack D, Buls N, Van Gompel G, Nieboer $\mathrm{KH}$, et al. : Dedicated sub $0.1 \mathrm{mSv}$ 3DCT using MBIR in children with suspected craniosynostosis : quality assessment. Eur Radiol 26 : 892899,2016

8. Jaffurs D, Denny A : Diagnostic pediatric computed tomographic scans of the head : actual dosage versus estimated risk. Plast Reconstr Surg 124 : 1254-1260, 2009

9. Kaasalainen T, Palmu K, Lampinen A, Reijonen V, Leikola J, Kivisaari R, et al. : Limiting CT radiation dose in children with craniosynostosis : phantom study using model-based iterative reconstruction. Pediatr Radiol 45 : 1544-1553, 2015

10. Kirmi O, Lo SJ, Johnson D, Anslow P : Craniosynostosis : a radiological and surgical perspective. Semin Ultrasound CT MR 30 : 492-512, 2009

11. Lenton KA, Nacamuli RP, Wan DC, Helms JA, Longaker MT : Cranial suture biology. Curr Top Dev Biol 66 : 287-328, 2005

12. Marchac D, Renier D : Craniosynostosis. World J Surg 13 : 358-365, 1989

13. Nagaraja S, Anslow P, Winter B : Craniosynostosis. Clin Radiol 68 : 284-292, 2013

14. Persing JA, Jane JA, Shaffrey M : Virchow and the pathogenesis of craniosynostosis : a translation of his original work. Plast Reconstr Surg 83 : 738-742, 1989

15. Regelsberger J, Delling G, Helmke K, Tsokos M, Kammler G, Kränzlein $\mathrm{H}$, et al. : Ultrasound in the diagnosis of craniosynostosis. J Craniofac Surg 17 : 623-625; discussion 626-628, 2006

16. Schweitzer T, Böhm H, Meyer-Marcotty P, Collmann H, Ernestus RI, Krauß J : Avoiding CT scans in children with single-suture craniosynostosis. Childs Nerv Syst 28 : 1077-1082, 2012

17. Schweitzer T, Kunz F, Meyer-Marcotty P, Müller-Richter UD, Böhm H, Wirth C, et al. : Diagnostic features of prematurely fused cranial sutures on plain skull X-rays. Childs Nerv Syst 31 : 2071-2080, 2015

18. Slater BJ, Lenton KA, Kwan MD, Gupta DM, Wan DC, Longaker MT : Cranial sutures : a brief review. Plast Reconstr Surg 121 : 170e-178e, 2008

19. Soboleski D, McCloskey D, Mussari B, Sauerbrei E, Clarke M, Fletcher A : Sonography of normal cranial sutures. AJR Am J Roentgenol 168 : 819-821, 1997

20. Soboleski D, Mussari B, McCloskey D, Sauerbrei E, Espinosa F, Fletcher A : High-resolution sonography of the abnormal cranial suture. Pediatr Radiol 28 : 79-82, 1998

21. Sze RW, Parisi MT, Sidhu M, Paladin AM, Ngo AV, Seidel KD, et al. : Ultrasound screening of the lambdoid suture in the child with posterior plagiocephaly. Pediatr Radiol 33 : 630-636, 2003

22. Vazquez JL, Pombar MA, Pumar JM, del Campo VM : Optimised lowdose multidetector CT protocol for children with cranial deformity. Eur Radiol 23 : 2279-2287, 2013

23. Weinzweig J, Kirschner RE, Farley A, Reiss P, Hunter J, Whitaker LA, et al. : Metopic synostosis : Defining the temporal sequence of normal suture fusion and differentiating it from synostosis on the basis of computed tomography images. Plast Reconstr Surg 112 : 1211-1218, 2003 